\title{
Access to oral rehydration solution and zinc supplementation for treatment of childhood diarrhoeal diseases in Sudan
}

\author{
Sagad Omer Obeid Mohamed ${ }^{*}$, Mansour Osman Alhaj Alawad, Asaad Ahmed Mohammed Ahmed \\ and Ahmed Abdallah Ali Mahmoud
}

\begin{abstract}
Objectives: The decline in diarrhoeal disease-related mortality globally has been attributed to the use of oral rehydration solution (ORS) and zinc supplementation. However, data on ORS and zinc supplementation in Sudan are scarce. We aimed to investigate the access to ORS and zinc treatments and the associated factors, through the analysis of the latest available data from Sudan-Multiple Indicator Cluster Survey (MICS)-2014 obtained from the United Nations Children's Fund (UNICEF).

Results: A total of 14,081 children were included in this analysis. During the 2 weeks preceding the survey, $29.3 \%$ of these children had a diarrhoeal disease. Only $18.9 \%$ and $14.8 \%$ of these children had received ORS and zinc supplements, respectively. Whereas children from the higher wealth index groups were more likely to receive ORS treatment (fourth group: $A O R=1.301 ; 95 \% \mathrm{Cl} 1.006-1.682$ ), children from rural areas were less likely to receive ORS treatment $(A O R=0.666 ; 95 \% \mathrm{Cl} 0.552-0.803)$ and zinc supplements ( $A O R=0.603 ; 95 \% \mathrm{Cl} 0.500-0.728)$. The results indicate the existence of unequal access to treatment of childhood diarrhoeal diseases among children under 5 years in Sudan.
\end{abstract}

Keywords: Diarrhoea, Children, ORS, Zinc, Epidemiology, MICS

\section{Introduction}

Diarrhoeal diseases create a global health burden with significant morbidity and mortality among children under 5 years in low and middle-income countries [1-3]. About half-a-million diarrhoeal disease-related deaths occur annually, with the highest rates of under- 5 year child diarrhoea-related mortality recorded in sub-Saharan Africa and South Asia [1, 4, 5]. Despite improvements in standards of living, diarrhoeal diseases still account for significant economic and social losses [6]. Eradication of most of the preventable deaths due to diarrhoeal diseases could be achieved by scaling up cost-effective interventions $[7,8]$.

*Correspondence: s.oom123@yahoo.com

Faculty of Medicine, University of Khartoum, Alqasr Avenue, P.O. Box 102, Khartoum, Sudan
The decline in the total diarrhoeal diseases-related mortality globally has been attributed to the increased use of oral rehydration solution (ORS), zinc supplementation, improved nutrition, hygiene, and sanitation [1013]. The World Health Organization (WHO) and United Nations Children's Fund (UNICEF) recommended ORS and zinc supplementation as a primary treatment for diarrhoeal diseases in children because ORS can reduce diarrhoeal deaths significantly and zinc supplementation can reduce the duration, severity, and recurrence of diarrhoeal diseases in children in the 2 to 3 months following its use [9-13].

Data on childhood diarrhoeal diseases and ORS and zinc supplementation in Sudan are scarce and most of the studies on diarrhoeal disease in Sudan were hospital or small-community-based studies. This study was conducted to investigate diarrhoeal diseases prevalence, 
access to ORS and zinc treatments, and their associated factors among under 5 years of age children in Sudan.

\section{Main text \\ Methods \\ Source of data}

In this study, we analysed the latest available data (5th round) of the Sudan-Multiple Indicator Cluster Survey (MICS), which was conducted from August to December 2014 at the national level, covering all 18 states of Sudan [14-16]. MICS was conducted by the Central Bureau of Statistics and Ministry of Health as part of the global MICS programme. Several international collaborators, including the WHO, World Food Programme (WFP), and the United Nations Population Fund (UNFPA), funded the Sudan-MICS [14-16]. UNICEF developed the global MICS programme in order to collect internationally comparable data on several indicators of the health condition of women and children. These data allow countries to generate conclusive evidence for use in policies and to monitor progress towards the internationally accepted commitments [14-16].

Participants of the MICS-2014 were recruited using a two-stage stratified clustered sampling method. The sample size for Sudan-MICS 2014 was calculated as 18,000 households, and the number of households occupied was 17,142 . Of these, 16,801 households were successfully interviewed. In the interviewed households, 14,751 children under 5 years were listed in the household questionnaires. The questionnaires were completed for 14,081 of them (response rate: 95.5\%). The questionnaires were pretested and administered to mothers or caretakers of the children. Further details regarding the MICS are published in reports elsewhere [14-16].

\section{Study variables}

The variables included in this study were selected based on the availability of the data collected. The study examined the following variables: child age (infants/1-2 year s/2-3 years/3-4 years/4-5 years); sex (boy/girl); area of residence (urban/rural); mother education (none/primary/secondary/higher); and household wealth index quintile (poorest/second/middle/fourth/richest). The primary outcome variables: a self-reported history of diarrhoea in the past 2 weeks (Yes/No), receiving ORS treatment (Yes/No), and receiving zinc supplementation (Yes/No).

\section{Data analysis}

The weighted analysis was conducted in two parts. First, we performed a bivariate regression analysis to examine associations between uptake of ORS, zinc treatment, and the selected associated factors. The selected significant variables ( $p$ value $<0.2)$ were further entered into the multiple logistic regression models and the results were reported as adjusted odds ratios (AOR), with a 95\% confidence interval. All statistical analyses were conducted using SPSS software version 20 (SPSS Inc., Chicago, IL, USA).

\section{Results}

The number of children under 5 years included in the analysis was 14,081 . Most of the participants (23.2\%) were in the 3-4-year age group, and $21 \%$ of participants were infants. Children from urban and rural areas were $27.4 \%$ and $72.6 \%$, respectively. The socio-demographic characteristics of the participants are presented in Table 1. From the analysis, 4088 (29.3\%) children reported having a diarrhoeal disease in the past 2 weeks. ORS and zinc supplements had been administered to 758 (18.9\%) and 593 (14.8\%) participants in this category respectively (Table 1$)$.

From the included children, the overall proportion of having a diarrheal illness in the past 2 weeks among under-5 year children was $29.3 \%$ (4088 patients). Treatment with ORS and zinc supplementation was provided

Table 1 Characteristics under-5 year children participated in the study

\begin{tabular}{|c|c|c|c|}
\hline Variable & Level & Total no & Percentages \\
\hline \multirow[t]{5}{*}{ Age group } & Infants & 2964 & 21.0 \\
\hline & 1-2 year & 2672 & 19.0 \\
\hline & 2-3 year & 2618 & 18.6 \\
\hline & 3-4 year & 3268 & 23.2 \\
\hline & 4-5 year & 2559 & 18.2 \\
\hline \multirow[t]{2}{*}{ Sex } & Male & 7157 & 50.8 \\
\hline & Female & 6924 & 49.2 \\
\hline \multirow[t]{2}{*}{ Area of residence } & Urban & 3862 & 27.4 \\
\hline & Rural & 10,219 & 72.6 \\
\hline \multirow[t]{4}{*}{ Mother education } & None & 5994 & 42.6 \\
\hline & Primary & 4936 & 35.1 \\
\hline & Secondary & 2152 & 15.3 \\
\hline & Higher & 982 & 7.0 \\
\hline \multirow[t]{5}{*}{ Wealth index } & Poorest & 3188 & 22.6 \\
\hline & Second & 3015 & 21.4 \\
\hline & Middle & 2956 & 21.0 \\
\hline & Fourth & 2684 & 19.1 \\
\hline & Richest & 2238 & 15.9 \\
\hline \multirow{2}{*}{$\begin{array}{l}\text { Having diarrhea in the } \\
\text { past } 2 \text { weeks }\end{array}$} & Yes & 4088 & 29.3 \\
\hline & No & 9878 & 70.7 \\
\hline \multirow[t]{2}{*}{ Received ORS } & Yes & 3251 & 81.1 \\
\hline & No & 758 & 18.9 \\
\hline \multirow[t]{2}{*}{ Received zinc } & Yes & 3416 & 85.2 \\
\hline & No & 593 & 14.8 \\
\hline
\end{tabular}


Table 2 Multiple logistic regression results of receiving ORS among under-5 year children

\begin{tabular}{llllll}
\hline Variable & Level & ORS (\%) & AOR & $\mathbf{9 5 \%} \mathbf{C l}$ & p value \\
\hline Age group & Infants & 15.7 & Ref & - & - \\
& 1-2 year & 20.6 & 1.379 & $(1.090-1.746)$ & 0.008 \\
& 2-3 year & 20.3 & 1.351 & $(1.057-1.728)$ & 0.016 \\
& 3-4 year & 20.5 & 1.413 & $(1.097-1.819)$ & 0.007 \\
Area of residence & 4-5 year & 16.6 & 1.068 & $(0.791-1.442)$ & 0.666 \\
\multirow{2}{*}{ Wealth index } & Urban & 23.8 & Ref & - & - \\
& Rural & 16.8 & 0.666 & $(0.552-0.803)$ & 0.000 \\
& Poorest & 14.9 & Ref & - & - \\
& Second & 18.2 & 1.234 & $(0.942-1.616)$ & 0.126 \\
& Middle & 20.3 & 1.296 & $(0.996-1.686)$ & 0.054 \\
& Fourth & 20.8 & 1.301 & $(1.006-1.682)$ & 0.045 \\
& Richest & 20.1 & 1.108 & $(0.822-1.493)$ & 0.502 \\
\hline
\end{tabular}

Table 3 Multiple logistic regression results of receiving zinc among under- 5 year children

\begin{tabular}{llllll}
\hline Variable & Level & Zinc (\%) & AOR & $\mathbf{9 5 \%} \mathbf{C l}$ & p value \\
\hline Area of residence & Urban & 19.6 & Ref & - & - \\
& Rural & 12.8 & 0.603 & $(0.500-0.728)$ & 0.000 \\
Mother educa- & None & 14.7 & Ref & - & - \\
tion & Primary & 13.6 & 0.860 & $(0.700-1.055)$ & 0.147 \\
& Secondary & 16.5 & 1.002 & $(0.775-1.295)$ & 0.987 \\
& Higher & 17.5 & 1.014 & $(0.718-1.431)$ & 0.937 \\
\hline
\end{tabular}

to $18.9 \%$ (758 patients) and $14.8 \%$ (593 patients) of the affected children, respectively (Table 1).

\section{Results of logistic regression analysis}

The binary regression analysis showed that access to ORT for diarrhoeal diseases was associated with the age group, area of residence, and wealth index quintiles. Multiple logistic regression analysis showed that children living in rural areas were less likely to receive ORS treatment than those in the urban areas $(\mathrm{OR}=0.666 ; 95 \% \mathrm{CI}$ $0.552-0.803)$, whereas children in the fourth $(\mathrm{OR}=1.301$; 95\% CI 1.006-1.682) wealth index group were more likely to receive ORS treatment than poorest wealth index group (Table 2). Regarding zinc treatment, multiple logistic regression results showed that access to zinc supplements was associated only with the area of residence. Children in rural areas were less likely to receive zinc supplements than those in the urban areas $(\mathrm{OR}=0.603$; 95\% CI 0.500-0.728) (Table 3).

\section{Discussion}

Most of the diarrhoeal-related mortality in children are due to dehydration and loss of electrolytes. Successful management of diarrheal diseases with ORS or increased fluid intake can prevent many of these deaths. The WHO and UNICEF started the integrated Global Action Plan for Pneumonia and Diarrhoea (GAPPD) in 2013 [17]. By 2025 , this programme seeks to reduce the incidence of diarrhoea by $75 \%$ among children under 5 years, as one of the specific objectives. However, this study revealed an increase from $27.8 \%$ prevalence recorded in 2000 [18]. This increase is cautionary and should draw the attention of stakeholders in the health sector in Sudan.

The analysis of data from the previous Sudan-MICS in 2000 revealed that age of a child, area of residence, and wealth index were significantly associated with the outcome [18], which is similar to the findings of the current study. Similar to the findings of other studies from Ethiopia and Iraq $[19,20]$, our findings indicate that children in younger age groups were more likely to have diarrhoeal diseases. The lower prevalence of diarrhoeal diseases in the oldest age group of these children may be due to acquired natural immunity [20].

In this study, the use of ORT was as low as $18.9 \%$. Similarly, ORS for treatment of diarrhoeal diseases in children has been reported to remain below $50 \%$ in many low-income countries, despite available evidence suggesting that scaling up the use of ORS is a cost-effective way to highly reduce preventable child deaths [21]. Children living in rural areas and were less likely to receive ORS treatment and zinc supplementation than those in the urban areas, whereas children in the higher wealth index groups were more likely to receive ORS treatment. Unequal access to treatment of the childhood diarrhoeal diseases among under 5 years children has also been reported in several developing countries [22, 23]. Household wealth has also been reported to be a determinant of the treatment-seeking behaviour for childhood diarrhoeal diseases [23].

Some high-burden countries have established successful scale-up programmes to increase coverage and access rates of ORS and zinc supplementation among target population groups $[8,24]$. Paediatric diarrhoeal treatment rates in Kenya increased from 0.8 to $15 \%$ in 5 years after permission was given to dispense zinc supplements over-the-counter and government procurement was shifted to co-packaged ORS and zinc. These policies created a competitive market for ORS and zinc supplements and enhanced healthcare provider practices in the management of diarrhoeal diseases [24].

\section{Limitations}

Due to limitations in the data obtained for this analysis, we could not include further analyses of other aspects of the treatment of diarrhoeal diseases such as increased home intake and feeding, antibiotic use for treatment of 
some the common infectious causes of diarrhoea, such as amoebic dysentery and giardiasis; and inpatient management of severe dehydration and shock. Further studies for the assessment of all of these aspects of diarrhoea treatment are highly recommended.

\author{
Abbreviations \\ MICS: Multiple indicator cluster survey; WHO: World Health Organization; \\ UNICEF: United Nations Children's Fund; UNFPA: United Nations Population \\ Fund; WFP: World Food Program; GAPPD: Global Action Plan for Pneumonia \\ and Diarrhoea.
}

\section{Acknowledgements}

The United Nations Children's Fund (UNICEF) is greatly acknowledged for making the data from the 2014 Sudan MICS available to us for analysis.

\section{Authors' contributions}

(SM) Involved in the conception of the research idea and data analysis; (SM, $M A, A A$, and $A M$ ) interpreted the results and drafted the manuscript. All authors revised the final manuscript. All authors read and approved the final manuscript.

\section{Funding}

No fund.

\section{Availability of data and materials}

The dataset used during this study is available from the corresponding author on reasonable request. The underlying dataset that was further analysed in this study is available from https://mics.unicef.org/surveys.

\section{Ethics approval and consent to participate}

Not applicable.

\section{Consent for publication}

Not applicable.

\section{Competing interests}

The authors declare that they have no competing interests.

Received: 10 June 2020 Accepted: 4 September 2020

Published online: 10 September 2020

\section{References}

1. Global Burden of Diarrheal Diseases Collaborators. Estimates of global, regional, national morbidity, mortality, and aetiologies of diarrheal diseases: a systematic analysis for the Global Burden of Disease Study 2015 Lancet Infect Dis. 2017;17:909-48.

2. Liu L, Chu Y, Oza S, et al. National, regional, and state-level all-cause and cause-specific under-5 mortality in India in 2000-15: a systematic analysis with implications for the Sustainable Development Goals. Lancet Glob Health. 2019;7(6):e721-e734734. https://doi.org/10.1016/S2214 $-109 \times(19) 30080-4$

3. O'Ryan G M, Ashkenazi-Hoffnung L, O'Ryan-Soriano MA, Ashkenazi S. Management of acute infectious diarrhea for children living in resourcelimited settings. Expert Rev Anti-infect Ther. 2014;12(5):621-32. https:// doi.org/10.1586/14787210.2014.901168.

4. GBD. Mortality and Causes of Death Collaborators Global, regional, and national life expectancy, all-cause and cause-specific mortality for 249 causes of death, 1980-2015: a systematic analysis for the Global Burden of Disease Study 2015. Lancet. 2015;2016(388):1459-544.

5. Sidoti F, Rittà M, Costa C, et al. Diagnosis of viral gastroenteritis: limits and potential of currently available procedures. J Infect Dev Ctries. 2015;9:551-61.

6. Mokomane M, Kasvosve I, de Melo E, Pernica JM, Goldfarb DM. The global problem of childhood diarrhoeal diseases: emerging strategies in prevention and management. Ther Adv Infect Dis. 2018;5(1):29-43. https ://doi.org/10.1177/2049936117744429.

7. Chopra M, Mason E, Borrazzo J, Campbell H, Ru-dan I, Liu L, Black RE, Bhutta ZA. End- ing of preventable deaths from pneumonia and diarrhea: an achievable goal. Lancet. 2013;381:1499-506. https://doi.org/10.1016/ S0140-6736(13)60319-0.

8. Lam F, Wentworth L, Cherutich P, Migiro S, Abdala K, Musyoka M, et al. An evaluation of a national oral rehydration solution and zinc scale-up program in Kenya between 2011 and 2016. J Glob Health. 2019;9(1):010505.

9. Black R, Fontaine $\mathrm{O}$, Lamberti $\mathrm{L}$, et al. Drivers of the reduction in childhood diarrhea mortality 1980-2015 and interventions to eliminate preventable diarrhea deaths by 2030. J Glob Health. 2019;9(2):020801. https://doi.org/10.7189/jogh.09.020801.

10. Florez ID, Al-Khalifah R, Sierra JM, et al. The effectiveness and safety of treatments used for acute diarrhea and acute gastroenteritis in children: protocol for a systematic review and network meta-analysis. Syst Rev. 2016;5(14):2016. https://doi.org/10.1186/s13643-016-0186-8.

11. Masanja $\mathrm{H}$, Mongi $\mathrm{P}$, Baraka J, et al. Factors associated with the decline in under five diarrhea mortality in Tanzania from 1980-2015. J Glob Health. 2019;9(2):020806. https://doi.org/10.7189/jogh.09.020806.

12. Choudhary TS, Sinha B, Khera A, et al. Factors associated with the decline in under-five diarrhea mortality in India: a LiST analysis. J Glob Health. 2019;9(2):020804. https://doi.org/10.7189/jogh.09.020804.

13. Lazzerini $M$, Wanzira $H$. Oral zinc for treating diarrhoea in children. Cochrane Database Syst Rev. 2016;12(12):CD005436. https://doi. org/10.1002/14651858.CD005436.pub5.

14. Surveys - UNICEF MICS. Mics.unicef.org. https://mics.unicef.org/surveys. Accessed 25 Nov 2019.

15. Central Bureau of Statistics (CBS), UNICEF Sudan. Multiple indicator cluster survey 2014 of Sudan, final report. Khartoum: UNICEF and Central Bureau of Statistics (CBS); 2016.

16. Central Bureau of Statistics (CBS), UNICEF Sudan. Multiple indicator cluster survey of fieldwork, key findings. Khartoum: UNICEF and Central Bureau of Statistics (CBS); 2014.

17. World Health Organization, Unicef. Ending preventable child deaths from pneumonia and diarrhea by 2025: the integrated Global Action Plan for Pneumonia and Diarrhea (GAPPD). Geneva: World Health Organization; 2013.

18. Siziya S, Muula AS, Rudatsikira E. Correlates of diarrhea among children below the age of 5 years in Sudan. Afr Health Sci. 2013;13(2):376-83. https://doi.org/10.4314/ahs.v13i2.26.

19. Getachew A, Guadu T, Tadie A, Gizaw Z, Gebrehiwot M, Cherkos DH, Menberu MA, Gebrecherkos T. Diarrhea prevalence and sociodemographic factors among under-five children in rural areas of North Gondar Zone, Northwest Ethiopia. Int J Pediatr. 2018. https://doi. org/10.1155/2018/6031594.

20. Siziya S, Muula AS, Rudatsikira E. Diarrhea and acute respiratory infections prevalence and risk factors among under-five children in Iraq in 2000. Ital J Pediatr. 2009;35:8. https://doi.org/10.1186/1824-7288-35-8.

21. Andrus A, Cohen R, Carvajal-Aguirre L, El Arifeen S, Weiss W. Strong community-based health systems and national governance predict improvement in coverage of oral rehydration solution (ORS): a multilevel longitudinal model. J Glob Health. 2020;10(1):010503. https://doi. org/10.7189/jogh. 10.010503.

22. Lee H-Y, Huy NV, Cho S. Determinants of early childhood morbidity and proper treatment responses in Vietnam: results from the Multiple Indicator Cluster Surveys, 2000-2011. Glob Health Action. 2016. https://doi. org/10.3402/gha.v9.29304.

23. Kawakatsu Y, Tanaka J, Ogawa K, Ogendo K, Honda S. Community unit performance: factors associated with childhood diarrhea and appropriate treatment in Nyanza Province, Kenya. BMC Public Health. 2017;17:202. https://doi.org/10.1186/s12889-017-4107-0.

24. Schroder K, Battu A, Wentworth L, Houdek J, Fashanu C, Wiwa O, et al. Increasing coverage of pediatric diarrhea treatment in high-burden countries. J Glob Health. 2019;9(1):0010503.

\section{Publisher's Note}

Springer Nature remains neutral with regard to jurisdictional claims in published maps and institutional affiliations. 